\title{
Spontaneous Crystallization of Light and Ultracold Atoms
}

\author{
S. Ostermann, F. Piazza, and H. Ritsch \\ Institut für Theoretische Physik, Universität Innsbruck, Technikerstraße 21, A-6020 Innsbruck, Austria
}

(Received 21 January 2016; published 24 May 2016)

\begin{abstract}
Coherent scattering of light from ultracold atoms involves an exchange of energy and momentum introducing a wealth of nonlinear dynamical phenomena. As a prominent example, particles can spontaneously form stationary periodic configurations that simultaneously maximize the light scattering and minimize the atomic potential energy in the emerging optical lattice. Such self-ordering effects resulting in periodic lattices via bimodal symmetry breaking have been experimentally observed with cold gases and Bose-Einstein condensates (BECs) inside an optical resonator. Here, we study a new regime of periodic pattern formation for an atomic BEC in free space, driven by far off-resonant counterpropagating and noninterfering lasers of orthogonal polarization. In contrast to previous works, no spatial light modes are preselected by any boundary conditions and the transition from homogeneous to periodic order amounts to a crystallization of both light and ultracold atoms breaking a continuous translational symmetry. In the crystallized state the BEC acquires a phase similar to a supersolid with an emergent intrinsic length scale whereas the light field forms an optical lattice allowing phononic excitations via collective backscattering, which are gapped due to the infinte-range interactions. The system we study constitutes a novel configuration allowing the simulation of synthetic solid-state systems with ultracold atoms including long-range phonon dynamics.
\end{abstract}

DOI: 10.1103/PhysRevX.6.021026

Subject Areas: Atomic and Molecular Physics, Condensed Matter Physics, Quantum Physics

\section{INTRODUCTION}

For a gas of pointlike particles off-resonantly illuminated by coherent light, the individual dipoles oscillate in phase, each emitting radiation in a characteristic pattern. When several particles contribute to the scattering, the corresponding amplitudes interfere, which leads to a strongly angle-dependent scattering distribution [1-3]. In addition, if the motional degree of freedom is relevant on the considered time scales, any high-field-seeking particle will be drawn towards the corresponding local light field maxima, where in turn light scattering is enhanced. This directional energy and momentum transfer between the gas and the field leads to an instability resulting in density fluctuations and potentially also in the formation of an ordered pattern. While for a room temperature gas this typically occurs only at very high pump powers [4-6], it can become important for very strong scatterers such as larger nano- or microparticles [7-13]. The stringent threshold conditions can be relaxed by laser cooling the gas to temperatures well below the $\mathrm{mK}$ range as well as by recycling the scattered light in optical resonators. In this

\footnotetext{
*stefan.ostermann@uibk.ac.at
}

Published by the American Physical Society under the terms of the Creative Commons Attribution 3.0 License. Further distribution of this work must maintain attribution to the author(s) and the published article's title, journal citation, and DOI. case, much weaker forces and thus lower light power are needed to create a substantial backaction effect of the scattered light onto the particles. This backaction was predicted to lead to rotonlike instabilities and spatial bunching even at moderate pump powers, as observed in several configurations [14-25].

A relevant question is thus whether these instabilities can in some cases lead to the formation of a stable crystalline phase in the steady state of such driven, dissipative systems. The first and simplest instance of such crystals is the self-ordered phase of transversally driven atoms in optical resonators [26-29], with the corresponding transition observable also as a quantum phase transition at zero temperature [30,31]. It has been shown recently that a similar phase is also realizable in longitudinally pumped ring cavities [32].

While this self-ordered phase shows some aspects shared by standard crystals such as a rotonlike mode [33], other characteristic features like the breaking of a continuous translational symmetry and a crystal spacing which is not externally fixed are both missing, since the resonator mirrors select a single electromagnetic mode. In order to include such features, one necessarily needs to couple the particles to several electromagnetic modes, ideally a continuum. This is the case in one-dimensional tapered optical nanofibers [34,35] or confocal cavities [36], where transversally driven atoms are predicted to spontaneously break the continuous symmetry into a crystal phase. 


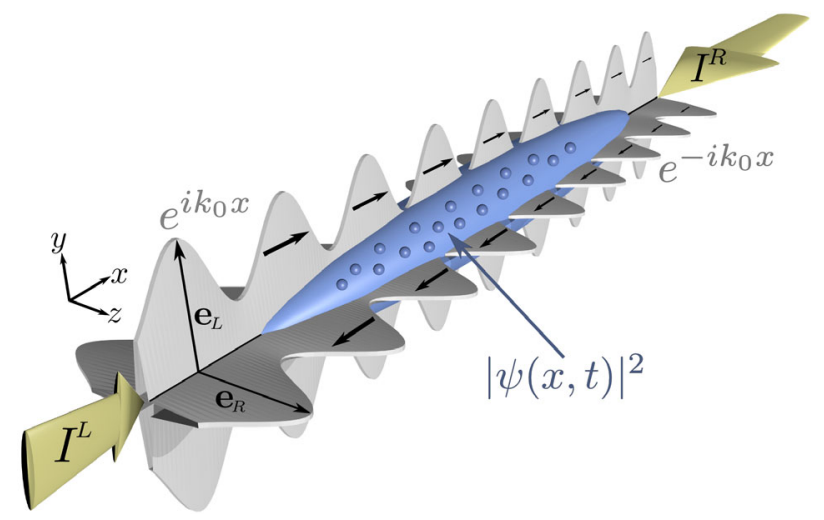

FIG. 1. Schematic representation of the considered setup. An elongated BEC interacting with two counterpropagating, noninterfering laser beams of orthogonal polarization. The two beams are far detuned from any atomic resonance in order to avoid mixing between the two polarizations. Both polarizations are assumed to be equivalent with respect to the considered atomic transition, the latter thus involving a spherically (or at least cylindrically) symmetric ground state. Alternatively to the use of two different polarizations, sufficiently different frequencies of the two counterpropagating lasers can be chosen.

The existence of a continuum of electromagnetic modes opens up the possibility for photons to crystallize, as it was studied with light propagating under electromagnetically induced transparency conditions through a nonlinear medium $[37,38]$.

In this work, we propose and characterize a novel crystalline phase of light and ultracold atoms. We consider a mirror symmetric and translation invariant setup as it is depicted in Fig. 1. It involves an elongated Bose-Einstein condensate (BEC) longitudinally illuminated by two counterpropagating Gaussian beams far detuned from any atomic resonance. The beams have either orthogonal polarization or a sufficiently large frequency difference to suppress any interference effects. Above a finite driving intensity, both atoms and light break a continuous translational symmetry leading to pattern formation with an intrinsically defined lattice spacing determined by the polarizability and density of the gas. The resulting state corresponds to a supersolid BEC trapped in an emerging optical lattice, the latter showing collective phononic excitations. The appearance of an emergent length scale in combination with lattice phonons-i.e., the appearance of a crystal of light-is a crucial difference from configurations where the drive is transverse to the direction in which the system organizes [34-36].

A useful property of the chosen geometry is that ample information about the coupled system dynamics can be retrieved from the reflected light fields in a completely noninvasive manner. The present study opens a new direction in (ultra)cold atom-lattice physics, naturally including long-range phonon-type interactions and real-time nondestructive monitoring.

\section{MODEL}

We consider a trapped atomic BEC interacting with the electromagnetic (EM) field driven by two far off-resonant, counterpropagating, orthogonally polarized laser beams, as depicted in Fig. 1. In the dispersive regime considered below, the EM field provides an optical potential for the BEC [see Eq. (1)], while the BEC significantly modifies the refractive index [see Eq. (3)]; thus, both field and matter are dynamical quantities.

The BEC is treated within the Gross-Pitaevskii (GP) mean-field approximation [39], whereby the condensate wave function satisfies the equation

$$
\begin{aligned}
i \hbar \frac{\partial}{\partial t} \psi(x, t)= & {\left[\frac{-\hbar^{2}}{2 m} \frac{\partial^{2}}{\partial x^{2}}+V(x)\right] \psi(x, t) } \\
& +\frac{g_{c} N}{A}|\psi(x, t)|^{2} \psi(x, t),
\end{aligned}
$$

where $m$ denotes the particle's mass, $g_{c}$ is the effective $s$-wave atom-atom interaction strength, and $N$ is the atom number. For computational simplicity, we assume the BEC to be confined by an extra transverse trapping potential $V_{\text {trap }}(x, y, z)$ such that the dynamics along the $y$ and $z$ axis is negligible. Therefore, the BEC wave function $\psi$ is assumed to be in the ground state of the transverse trap with characteristic size $d_{y}=d_{z}=\sqrt{A}$, where $A$ denotes the BEC cross section. Such a quasi-one-dimensional treatment is eligible if the BEC's chemical potential $\mu$ is much smaller than the characteristic transverse trap frequency: $\mu \ll \hbar \omega_{y, z}$. The wave function satisfies the normalization condition: $\int d x|\psi(x, t)|^{2}=1$.

The total optical potential for the BEC has two contributions:

$$
V(x)=V_{\text {trap }}(x)+V_{\text {opt }}(x),
$$

representing the static trapping potential $V_{\text {trap }}$ and the longitudinal (along $x$ ) optical potential $V_{\text {opt }}$ determined by the dynamical part of the injected and scattered EM field [see Eq. (5)]. The latter consists of two far off-resonant fields with orthogonal polarizations driven from the left $(L)$ and right $(R)$ side of the BEC, as depicted in Fig. 1. The two polarization components of the field satisfy the Helmholtz Eq. (3).

The atoms inside the BEC are described as linearly polarizable particles with a scalar polarizability $\alpha$ where the imaginary part is negligibly small; i.e., spontaneous emission of the atoms is neglected. This corresponds to the assumption that the driving laser frequency $\omega_{l}$ is sufficiently far detuned form any atomic resonance to prevent substantial internal excitation. This avoids spontaneous emission and thus mixing of the two counterpropagating EM components via Raman scattering, as it is used for near-resonant polarization gradient cooling, may be neglected. 
While for spin-polarized atoms the polarizability is field direction dependent in general, we assume the same polarizability for both polarizations orthogonal to the laser axis being the quantization axis. This corresponds to transitions from a spherically (or at least cylindrically) symmetric atomic ground state. The impinging laser fields from the left and right are approximated by plane waves so that we can write the EM field components as $\mathbf{E}_{L, R}(x, t)=\left[E_{L, R}(x) e^{i \omega_{l} t}+\right.$ c.c. $] \mathbf{e}_{L, R}$, with the orthogonality condition $\mathbf{e}_{L} \cdot \mathbf{e}_{R}=0$. As the light transit time through the sample is negligible compared to all other time scales, the propagation delay of the EM field is adiabatically eliminated and the two field envelopes ( $L$ for the field from left and $R$ for the field from right) satisfy the Helmholtz equations

$$
\frac{\partial^{2}}{\partial x^{2}} E_{L, R}(x)+k_{0}^{2}[1+\chi(x)] E_{L, R}(x)=0
$$

with the wave number $k_{0}$ of the incoming beams and the susceptibility $\chi(x)$ of the BEC. This susceptibility depends on the condensate's density and is given by

$$
\chi(x)=\frac{\alpha N}{\epsilon_{0} A}|\psi(x)|^{2},
$$

where $\psi(x, t)$ is the solution of Eq. (1). The directionality of the field propagation in the Helmholtz equations [Eq. (3)] is defined by the boundary conditions, according to which the $L$ component has a finite imposed amplitude on the left end of the system and the $R$ component has such on the right end (see also Appendix B).

As soon as one knows the spatial distribution of the electric fields, one can calculate the optical potential for the atoms via

$$
V_{\mathrm{opt}}(x)=-\frac{\alpha}{A}\left[\left|E_{L}(x)\right|^{2}+\left|E_{R}(x)\right|^{2}\right] .
$$

Inserting the optical potential Eq. (5) into Eq. (1) leaves us with the set of three coupled differential equations: the GP Eq. (1) and the two Helmholtz equations [Eq (3)], describing the nonlinear dynamics of our system. The degree of nonlinearity resulting from the atom-light coupling is quantified by the dimensionless constant $\zeta$ defined as

$$
\zeta:=\frac{\alpha N}{\varepsilon_{0} \lambda_{0} A}=\frac{\alpha}{\epsilon_{0}} n \frac{L}{\lambda_{0}},
$$

where $n=N / A L$ is the three-dimensional density of the homogeneous BEC with $L$ its characteristic extension along $x$. Because of the adiabatic approximation involved in the Helmholtz equation, the EM fields depend only parametrically on time through the dynamical refractive index set by the BEC density.
Because of the orthogonality of the two chosen polarizations there is no interference between the two counterpropagating components of the EM fields. Therefore, the optical potential [Eq. (5)] depends only on the absolute value squared of the fields. This important feature guarantees the translation invariance of the setup along the $x$ direction nevertheless maintaining a mirror symmetric setup. Indeed, since we are driving with plane-wave lasers, as long as the BEC density is homogeneous, the EM fields $E_{L, R}(x)$ in Eq. (3) are also plane waves, leading to a translation invariant optical potential Eq. (5). This invariance with respect to continuous translations is spontaneously broken above a finite driving intensity, as we discuss in Sec. III. In the resulting crystalline phase, the lattice constant is intrinsically established, as we discuss in Sec. IV. This is due to the fact that no specific modes are selected and the fields can counterpropagate independently.

\section{DYNAMICAL INSTABILITY TOWARDS CRYSTALLIZATION}

As already mentioned above, due to the orthogonality of the polarizations of the two injected counterpropagating laser fields, the particles do not feel any longitudinal optical forces. Naively, one could thus expect the BEC to remain unperturbed independently of the pump intensity. In this section, we show that this is actually not the case, as above a particular threshold driving strength small density fluctuations lead to backscattering of light, which in turn amplifies these fluctuations. This leads to an instability towards crystallization in the longitudinal direction. The latter can be described by considering the collective excitation spectrum of the system for a spatially homogeneous density distribution of the BEC, $\psi_{0}(x, t)=1 / \sqrt{L}$, with the corresponding propagating field solution of Eq. (3). These are plane waves of the form $E_{L, R}^{(0)}=C \exp \left( \pm i k_{\text {eff }} x\right)$, with the modified wave number

$$
k_{\mathrm{eff}}=\frac{2 \pi}{\lambda_{0}} \sqrt{1+\zeta \lambda_{0}\left|\psi_{0}\right|^{2}}=\frac{2 \pi}{\lambda_{0}} \sqrt{1+\frac{\alpha}{\epsilon_{0}} n}
$$

where $C$ is a real number fixed by the driving strength.

The spectrum is obtained by linearizing the coupled equations [Eqs. (1) and (3)] with the ansatz $\psi=$ $\left(\psi_{0}+\delta \psi\right) e^{-i \mu t}$ and $E_{L, R}=E_{L, R}^{(0)}+\delta E_{L, R}$. Here, $\delta \psi$ and $\delta E$ are small deviations from the stationary solutions $\psi_{0}$ or $E_{L, R}^{(0)}$ and $\mu$ is the BEC chemical potential (see Appendix A for details). This yields

$\hbar^{2} \omega_{q}^{2}=\frac{\hbar^{2} q^{2}}{2 m}\left[\frac{\hbar^{2} q^{2}}{2 m}+2 g n-\frac{64 \pi^{2} A \zeta^{2}}{c N L} \frac{1}{q^{2}-4 k_{\mathrm{eff}}^{2}} I^{L, R}\right]$.

Here, $I^{L, R}$ denotes the intensity (in $\mathrm{W} / \mathrm{m}^{2}$ ) of the incoming light, which we have chosen to be equal from the left and right. 


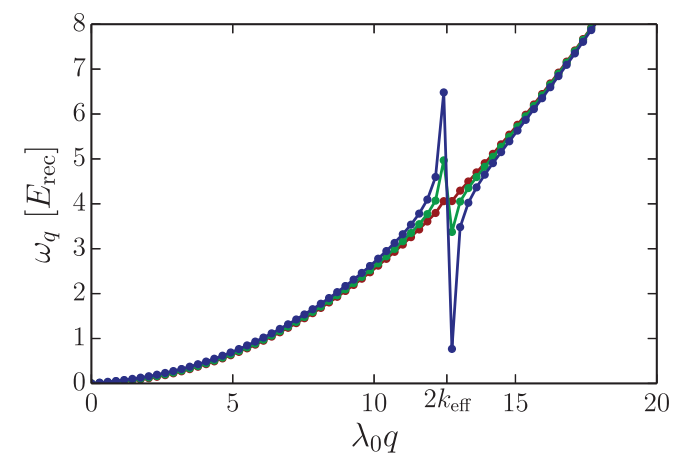

FIG. 2. Excitation spectrum Eqs. (8)] in the homogeneous phase for different field intensities: $I^{L, R}=2.0$ (red), $I^{L, R}=20.0$ (green), and $I^{L, R}=60.0$ (blue) $\left(\zeta=0.1, L=100 \lambda_{0}, g_{c} N / A \lambda_{0}=E_{\text {rec }}\right)$.

The above analytical expression Eq. (8) is very useful to understand some essential features of the atom-light interaction in the present setup and, in particular, the nature of the crystallization transition. Apart from the last term, we recognize the known form of the Bogoliubov spectrum of interacting BECs [39], with the linear-in- $q$ behavior corresponding to phononic excitations at low $q$. The last term, on the other hand, is the only one resulting from the atom-light interactions. The first thing to note is that its denominator vanishes at $q= \pm 2 k_{\mathrm{eff}}$, which tells us immediately that the modified wave number Eq. (7) sets the favored momentum for the appearance of the instability. However, the vanishing of the denominator is compensated by the diverging BEC length $L$, at every finite atom number $N$ (note that $\zeta \sim N$ ). The limit $L \rightarrow \infty$ of Eq. (8) actually has to be taken, since the stationary plane-wave solution $E_{L, R}^{(0)} \sim \exp \left( \pm i k_{\text {eff }} x\right)$ about which we linearize only makes sense for a homogeneous and infinite atomic medium, so that the edges may be neglected. This indeed allows us to neglect the reflection of the incident wave by the change in refractive index at the BEC edges. Such finite-size effects, included in the numerical solutions we describe in Sec. IV, become irrelevant for large systems, as we demonstrate below.

One way to obtain the proper result for Eq. (8) in the limit $L \rightarrow \infty$ is to consider that for any finite $L$ the allowed momenta $q$ take only quantized values as multiples of $2 \pi / L$. Before taking the limit $L \rightarrow \infty$ it is instructive to compute the spectrum Eq. (8) for fixed finite $L$, as we show in Fig. 2 for different values of $I^{L, R}$. One recognizes a gap opening at $q=2 k_{\text {eff }}$ for any finite $I^{L, R}$, i.e., any finite driving strength. The spectrum develops a minimum at the finite momentum $q=2 k_{\text {eff }}+(2 \pi) / L$, which corresponds to a roton minimum in the language commonly adopted for standard crystal formation [40]. It constitutes a generalization to continuous-symmetry breaking of the rotonlike instability observed with a BEC in an optical cavity [33]. In a similar manner as in standard crystals, the crystallization threshold can be calculated by finding the drive intensity at which the roton energy approaches zero. This leads to the threshold condition $\omega_{2 k_{\text {eff }}+(2 \pi) / L}=0$. We are now in the

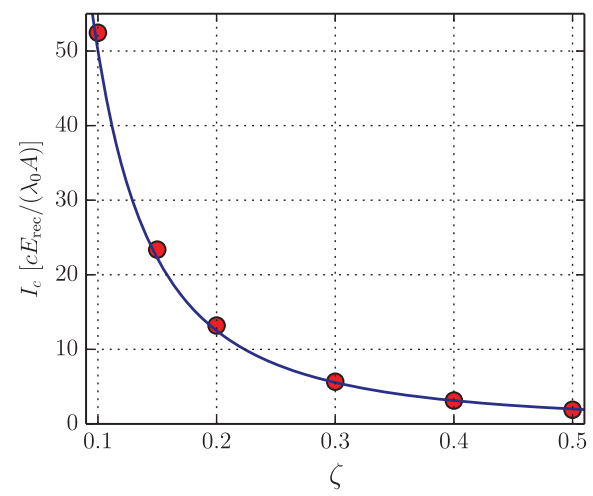

FIG. 3. $\zeta$ dependence of the critical intensity. The solid blue line depicts the analytical result defined by Eq. (9), whereas the red dots depict numerical threshold estimations for large system sizes $\left(L=120 \lambda_{0}\right)\left(\zeta=0.1, g_{c} N / A \lambda_{0}=E_{\text {rec }}\right)$.

position to take the limit $L \rightarrow \infty$. In doing this, we note that we have to keep the atom number $N$ constant in order to get a finite critical drive strength. Otherwise, if we perform the standard thermodynamic limit $N / L=$ const, the energy of the system diverges and the crystallization threshold vanishes. This divergence is an artifact of our model in which the light-mediated atom-atom interaction is of infinite range since the EM field is adiabatically adapting to the BEC configuration. The inclusion of the dynamics of the EM field (retardation effects) would introduce a finite range and thus eliminate the divergence in the energy. Still, the resulting range is expected to be larger than the typical BEC size $L$ so that our calculation should be valid for any realistic system size. Taking the $L \rightarrow \infty$ limit we thus get the critical driving intensity

$$
I_{c}^{L, R}=\frac{c E_{\mathrm{rec}} N}{\lambda_{0} A} \frac{1}{\zeta^{2}}=c E_{\mathrm{rec}} \frac{\varepsilon_{0}^{2}}{\alpha^{2}} \frac{1}{n} \frac{\lambda_{0}}{L},
$$

where we introduce the recoil energy, $E_{\text {rec }}:=\hbar \omega_{\text {rec }}=$ $\hbar^{2} k_{0}^{2} /(2 m)$.

Note that in the $L \rightarrow \infty$ limit with constant $N$ the BEC becomes more and more dilute, which renders the direct atom-atom coupling $\sim g_{c}$ eventually irrelevant. In Fig. 3, the analytical expression Eq. (9) is compared with numerically estimated thresholds for large system sizes (see Sec. IV). We find full agreement between the linear instability threshold and the numerical threshold found by studying the imaginary time evolution of Eqs. (1) and (3). This numerical approach to finite-sized systems is described next.

\section{CRYSTAL OF LIGHT AND ATOMS}

After showing that the homogeneous system is unstable above a certain driving intensity, we show that a stable crystalline phase is reached and study its properties by numerically solving the coupled GP [Eq. (1)] and Helmholtz [Eq. (3)] equations. We perform an imaginary 


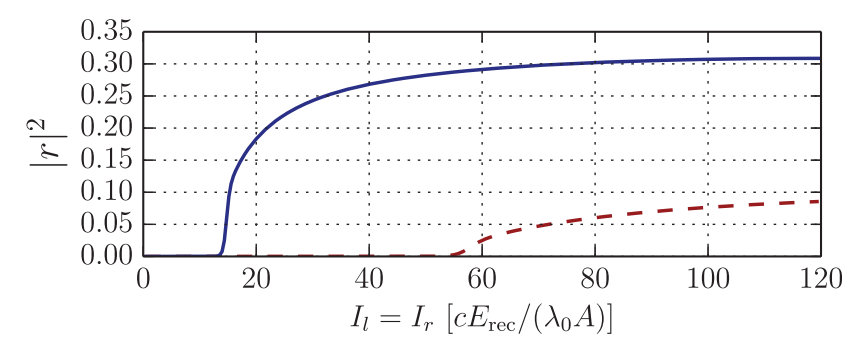

FIG. 4. Dependence of the reflection coefficient of the BEC on the incoming field amplitudes for different atom-field couplings $\zeta=0.1$ (dashed red line) and $\zeta=0.2$ (solid blue line). The remaining parameters are the same as in Fig. 2.

time evolution of the system Eqs. (1)-(3), i.e., replace $t \rightarrow i \tau$, which yields the ground state of the system for long enough evolution times. For a detailed description of the numerical methods, see Appendix B.

To determine the crystal transition point as a function of driving intensity, we compute the total reflectivity of the BEC with respect to the intensity of either one of the incident beams, which we again take to be equal. For large enough system sizes, a clear threshold behavior is visible at a critical driving intensity, whereby the reflectivity grows from essentially zero with almost infinite slope, cf. Fig. 4. The hereby found critical intensity is in perfect agreement with the analytical result(see Fig. 3). As we mention in the previous section, finite-size effects manifest due to the presence of the edges of the BEC. In the calculations described in this section and in Sec. VI, there is no further trapping potential along $x$ and the BEC is confined within a box of size $L$, so that the BEC has sharp edges for the light impinging at $x=0$ and $x=L$ (see Appendix B for more details). In Sec. VII, we add an harmonic trap along $x$ and show that the qualitative behavior is the same as that described here. The BEC edges create a quick increase of the refractive index, which induces a small amount of reflection of the incoming beam. As apparent from Fig. 4, this reflection is irrelevant for large system sizes compared to the reflection present in the crystalline phase.

The large light reflection above threshold is due to the appearance of a large spatial modulation of the BEC, forming the density grating shown in Fig. 5(a). This corresponds to a continuous-symmetry breaking at the threshold leading to a crystalline phase, which for the phase-coherent BEC implies supersolid order. Each peak in the density grating reflects the incoming light, resulting in a damped modulation of the intensity of each polarization component across the condensate, as shown in Fig. 5(b). While the modulation of each component's intensity $I^{L, R}$ is damped across the system, the modulation of the total intensity $I^{\text {tot }}=I^{L}+I^{R}$ is not damped, resulting in a periodic optical-lattice potential for the BEC, which matches its density grating.

An important feature of the optical lattice emerging in the crystalline phase is the intrinsic character of the lattice

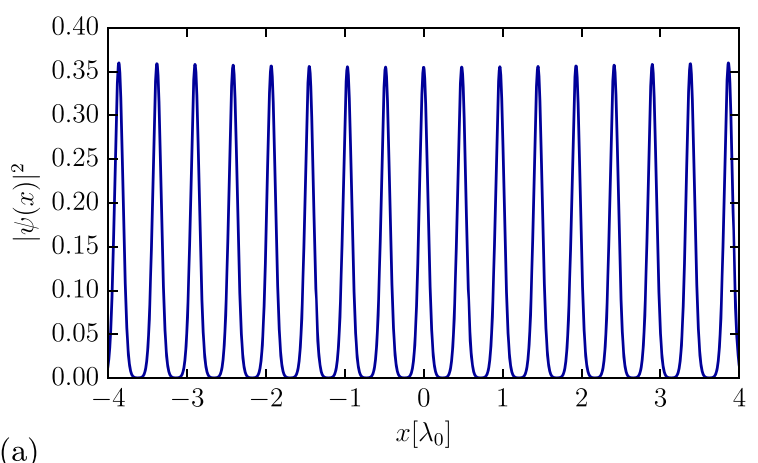

(a)

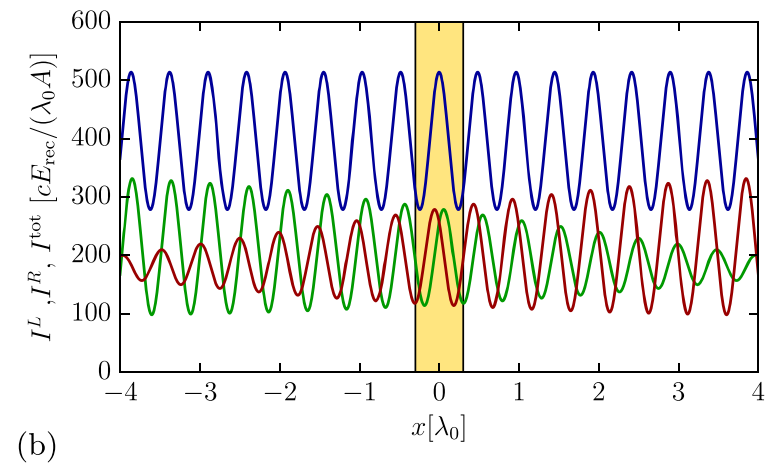

FIG. 5. (a) Crystal ground state for $\zeta=0.1, I_{l}=I_{r}=200$ and (b) corresponding intensity distribution for the field from left (green line) and right (red line). The solid blue line depicts the sum of both intensities. A zoom into the yellow shaded region can be found in Fig. 8. The remaining parameters are $g_{c} N / A \lambda_{0}=E_{\mathrm{rec}}$ and $L=10 \lambda_{0}$.

spacing, which is not fixed externally but rather set by the BEC density and atom polarizability. This is a clear difference with respect to the self-ordering in optical resonators, where the spacing is externally fixed by the cavity mirrors [26], and also to the case of self-ordering of transversally driven atoms coupled to the continuum of modes of optical fibers, where the spacing is fixed by the driving frequency and fiber dispersion [34,35]. As we anticipate in Sec. III, the appearance of the rotonlike instability at the characteristic momentum $2 k_{\text {eff }}$ leads to the following prediction for the emergent lattice spacing:

$$
d=\frac{\pi}{k_{\mathrm{eff}}}=\frac{\lambda_{0}}{2 \sqrt{1+\frac{\zeta \lambda_{0}}{L}}}=\frac{\lambda_{0}}{2 \sqrt{1+\frac{\alpha}{\epsilon_{0}} n}} .
$$

The emergent spacing is always smaller than the one in vacuum $\lambda_{0} / 2$. This feature can be qualitatively reproduced also within a toy model, where the medium is approximated by a set of beam splitters [41]. This typically small but nonetheless crucial effect is also present when using counterpropagating beams with equal polarization and is essential for atom trapping in optical lattices [42]. If the atoms were indeed trapped with the vacuum spacing $\lambda_{0} / 2$, the EM field would be perfectly reflected and no standing 
wave could actually be formed and thus no trapping would be possible. It is only through the slight renormalization $d<\lambda_{0} / 2$ that perfect reflection is avoided. What our scheme with orthogonally polarized counterpropagating beams allows is to make the small renormalization of $d$ coincide with the appearance of a large density modulation out of a homogeneous phase, i.e., a crystallization.

The existence of an intrinsic lattice spacing in the crystalline phase implies, as well, the presence of phononic excitations of the lattice, as we discuss in the next section.

\section{EXCITATIONS OF THE CRYSTAL: PHONONS}

Further insight into the properties of the atom-light crystal is provided by analyzing its excitation spectrum. As done in Sec. III, we linearize the coupled system of Eqs. (1) and (3). However, now the perturbation is performed around the symmetry-broken stationary solution. The result is presented in Fig. 6 for a driving intensity slightly above threshold. Details of the calculation are given in Appendix A. Since translation invariance is broken, the matrices describing the linear system are not diagonal in momentum space requiring a discretization of the position (momentum) continuum. Moreover, while the total light intensity and atom density are periodic, the intensity of each polarization component is not, due to accumulated reflection along the density grating, introducing the decaying envelope shown in Fig. 5(b). This prevents the use of the quasimomentum to label the excitation modes.

In Fig. 6, we label the eigenvalues based on their dominant momentum component $q_{\max }$, extracted from the corresponding eigenvector. This allows us to split the spectrum into three regions separated by gaps at $q_{\max }=k_{\text {eff }}$ and $q_{\max }=2 k_{\text {eff }}$.

The gap at $q_{\max }=k_{\text {eff }}$ opens up for $I^{L, R}>I_{c}^{L, R}$ due to the appearance of an optical lattice potential for the atoms with a $\pi / k_{\text {eff }}$ periodicity. It separates the two bands which, slightly above threshold, are characterized by eigenvectors with a clearly dominant momentum component (see left and middle insets in Fig. 6).

On the other hand, the gap at $2 k_{\text {eff }}$ is the same one appearing in the homogeneous phase (see Fig. 2). As we discuss in Sec. III, at the critical drive intensity $I_{c}^{L, R}$ the gap is such that the energy of the mode with momentum $q=2 k_{\text {eff }}+2 \pi / L$ (momentum is still a good quantum number for $\left.I^{L, R} \leq I_{c}^{L, R}\right)$ vanishes. Out of this zero-energy mode at $2 k_{\text {eff }}$ (not resolved with the discretization of Fig. 6), and beyond the critical point $I^{L, R}>I_{c}^{L, R}$, the lattice-phonon branch develops for $q_{\max }>2 k_{\text {eff }}$. The momentum distribution of the lattice-phonon eigenvectors is characterized by the splitting of the single peak at $2 k_{\text {eff }}$ into two neighboring peaks (see rightmost inset of Fig. 6). The phonon wavelength is set by the distance between the two nearby maxima appearing in the momentum distribution. This generates the slow beating in coordinate space.

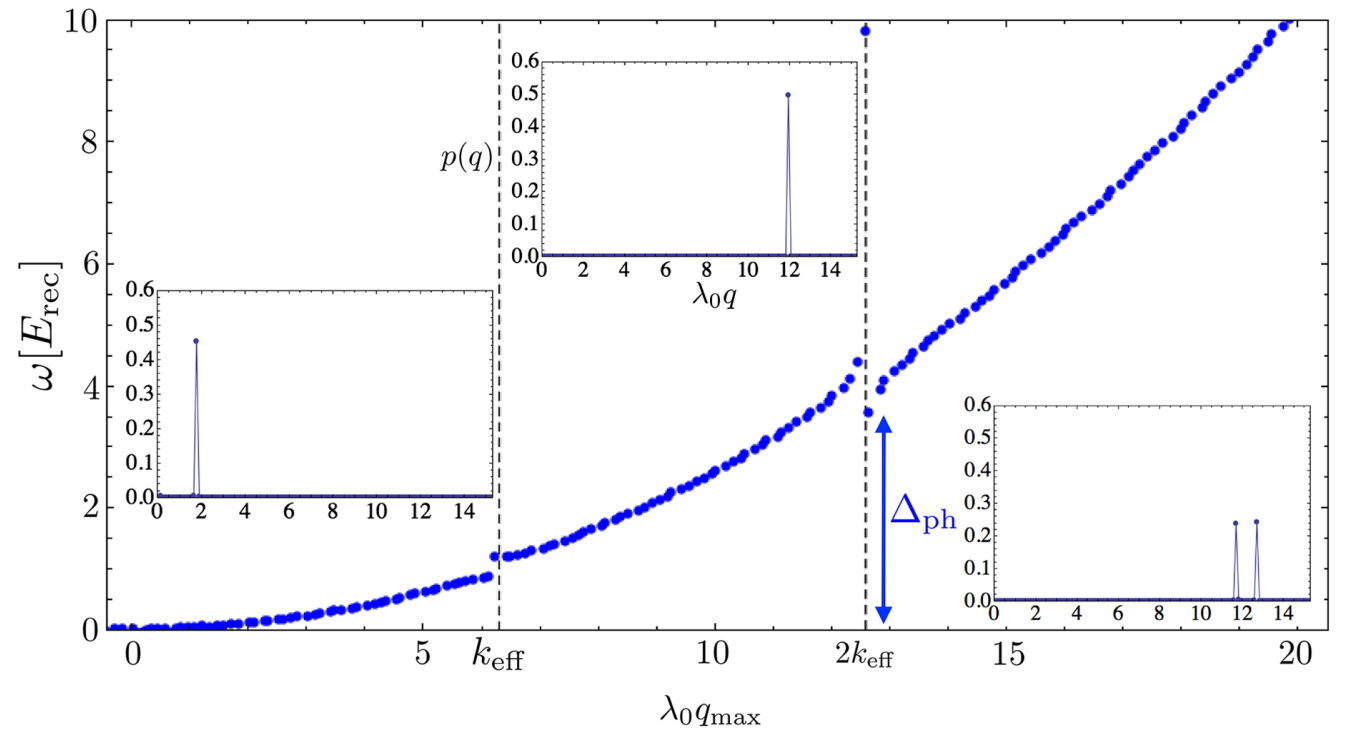

FIG. 6. Excitation spectrum of the atom-light crystal. The blue points are the eigenvalues of the GP and Helmholtz equations linearized about the crystalline stationary state (see Appendix A). The numerical diagonalization is performed with a momentum-space discretization $d q=2 \pi / L$. The parameters are the same as in Fig. 2 except for $L=50$ and a fixed drive intensity $I^{L, R}=50$ (slightly above threshold). $q_{\max }$ is the momentum corresponding to the largest component of the eigenvector of each eigenvalue. The insets show examples of eigenvectors (unormalized probability in momentum space) for three different eigenvalues representative of each region of the spectrum, from left to right: $\lambda_{0} q_{\max }=1.38<\lambda_{0} k_{\text {eff }}, \lambda_{0} k_{\text {eff }}<\lambda_{0} q_{\max }=11.9<2 \lambda_{0} k_{\text {eff }}$, and $\lambda_{0} q_{\max }=12.7>2 \lambda_{0} k_{\text {eff }}$. The latter region corresponds to lattice phonons, characterized by two symmetric pairs of peaks about a finite momentum. This phononic branch $q_{\mathrm{max}}>2 k_{\text {eff }}$ has a gap $\Delta_{\mathrm{ph}}$. Its analytical estimate in Eq. (11) yields $\Delta_{\mathrm{ph}} \simeq 2 \sqrt{2} E_{\mathrm{rec}}$, in reasonable agreement with the numerical data. 
With a finite system size $L$, the longest wavelength is of the order of $L$.

Moreover, the lattice-phonon branch is gapped, in the sense that its lowest energy mode at $q_{\max }$ slightly above $2 k_{\text {eff }}$ has a finite energy, as visible in Fig. 6. More importantly, this gap remains finite in the thermodynamic limit $L \rightarrow \infty$. We can estimate the size of the latticephonon gap close to threshold by using Eq. (8) and computing the energy of the mode next to the zero-energy mode. This yields

$$
\Delta_{\mathrm{ph}}^{2} \simeq 4 \frac{\hbar^{2} k_{\mathrm{eff}}^{2}}{2 m}\left(2 \frac{\hbar^{2} k_{\mathrm{eff}}^{2}}{2 m}+g n\right),
$$

which takes the value $\Delta_{\mathrm{ph}}^{2} \simeq 8 E_{\mathrm{rec}}^{2}$ in the thermodynamic limit $L \rightarrow \infty$ with $N=$ const. As we discuss in Sec. III, in this limit $I_{c}^{L, R}$ remains finite while $n \rightarrow 0$ and $k_{\text {eff }} \rightarrow k_{0}$. Another choice of thermodynamic limit is possible: $L, N \rightarrow \infty$ with $n=$ const, where $I_{c}^{L, R} \rightarrow 0$ and the gap is still given by Eq. (11). The existence of an energy gap for lattice phonons is due to the long-range nature of the interactions, as it can be already predicted within a classical model of interacting pointlike particles [43]. From a more general field-theoretical perspective, some of the gapless Goldstone modes expected from the continuous-symmetry breaking can indeed disappear (i.e., become gapped) due to the long range of the interactions, as it, for instance, happens to the longitudinal phonons of a three-dimensional Wigner crystal [44]. As long as retardation effects can be neglected, our interactions will be infinite-ranged, the lattice phonons gapped, and thus quantum or thermal fluctuations will not destroy crystalline order even in truly one dimension [45].

The existence of lattice phonons among the collective excitations is confirmed by numerical simulations of the real-time dynamics of the system, as we describe in the next section.

\section{CRYSTALLISATION DYNAMICS AFTER A QUENCH}

In this section, we investigate the real-time dynamics of the system by directly solving Eqs. (1) and (3). This allows us to analyse the crystallization dynamics after a sudden turn-on (quench) of the pump laser strength from zero to a value above threshold at $t=0$. The corresponding time evolution of the BEC reflectivity, kinetic energy, as well as the evolution of the BEC density and total light intensity are shown in Figs. 7 and 9.

As is apparent from the behavior of the reflectivity and kinetic energy $E_{\text {kin }}(t)=\int d x \hbar^{2}\left|\partial_{x} \psi\right|^{2} / 2 m$, the crystalline order is reached after a few inverse recoil frequencies, after which both quantities perform oscillations about a finite value. These residual oscillations are triggered by the energy gained by the system upon forming the density grating together with the optical lattice. The reason that this

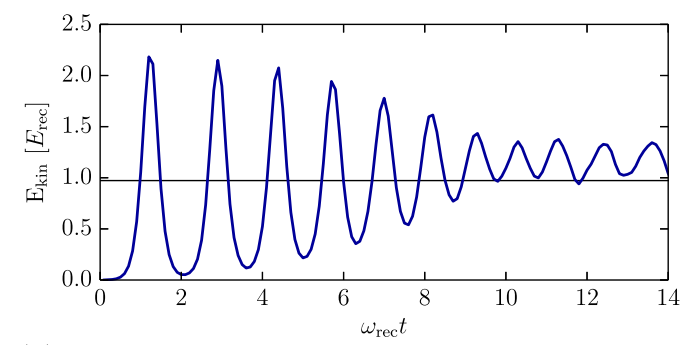

(a)

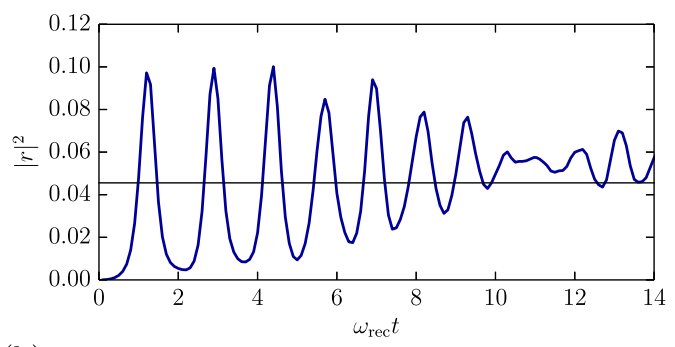

(b)

FIG. 7. (a) Real-time evolution of the kinetic energy for $\zeta=0.1, I_{l}=I_{r}=100, g_{c} N=1$. (b) Real-time evolution of the reflection coefficient for the same parameters as in (a). The solid black line shows the mean value of the corresponding functions.

effect takes on a prominent role in the studied case is found by looking at Fig. 8, which shows the zoom into two peaks of the intensity distribution of the crystal. One recognizes that the maxima of the intensity distributions of the two fields coming from the left and right (blue dots in Fig. 8) do not coincide with the maximum of the total intensity distribution (black dot in Fig. 8) at which the atoms are trapped. Therefore, the trapped atoms feel a strong field gradient for each single component because they do not sit at the maxima of the two counterpropagating fields, as

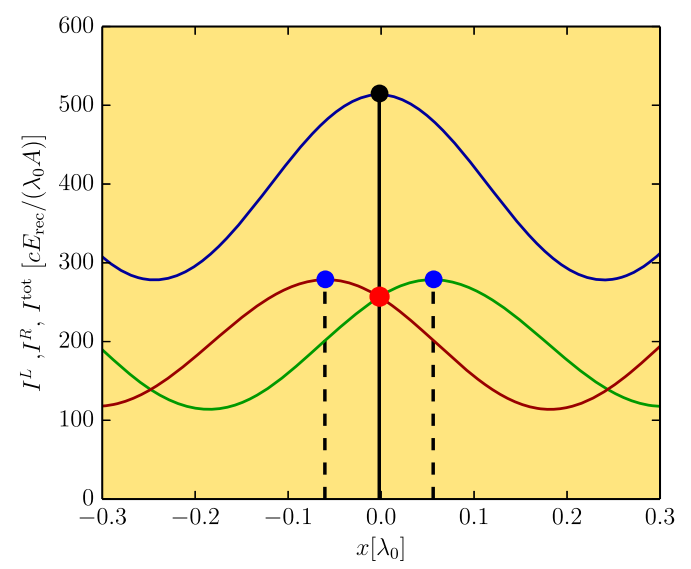

FIG. 8. Zoom into the yellow shaded region of Fig. 5. The blue dots mark the maxima of the field from left (green line) and right (red line), whereas the black dot marks the maximum of the total field intensity (blue line). The red dot shows the actual position of the particles. 


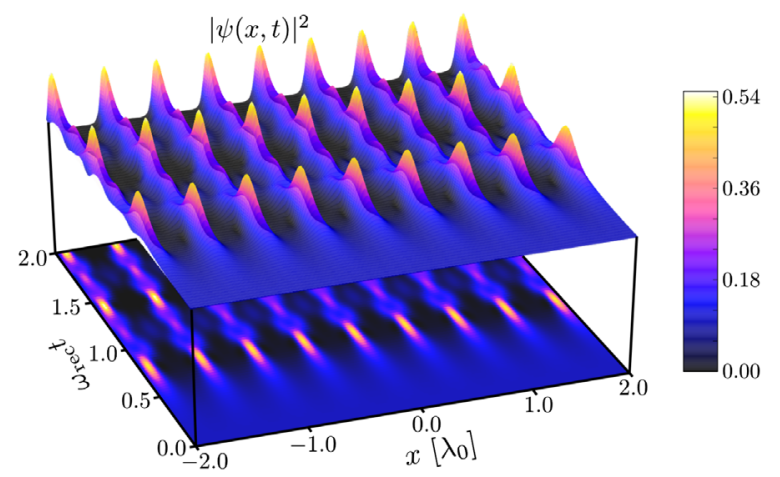

(a)

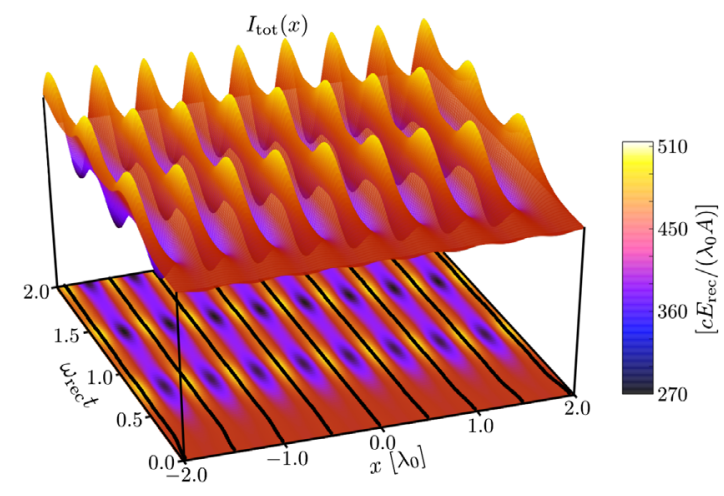

(b)

FIG. 9. Real-time dynamics of the (a) BEC density distribution and (b) the total light intensity for the same parameters as in Fig. 5. The solid black lines in (b) show the time evolution of the intensity maxima.

would be, for example, the case in optical lattices. This leads to a large coupling between the two counterpropagating fields and the atoms, leading to strong long-range interactions inducing collective excitations.

The corresponding dynamics of the BEC density and the total light intensity are shown in Fig. 9. As one can see from the solid lines marking the evolution of the intensity maxima, they start at a lattice spacing of $\lambda_{0} / 2$ and move closer together in time, reaching the emergent spacing $d$. In addition, we see the presence of residual oscillations about the crystalline order. In particular, the light intensity shows both compression modes, modulating the amplitude of the optical lattice in time, and phonons, modulating the spacing. The latter are clearly visible from the dynamics of the intensity maxima shown in Fig. 10. Since we are neglecting retardation of the fields, the energy can be redistributed among the collective degrees of freedom but not dissipated. Initially, for $\omega_{\text {rec }} t \sim 1$, mostly compression modes are excited. Subsequently, part of the energy stored in the compression modes is transferred to lattice phonons for $\omega_{\text {rec }} t \gtrsim 5$. In Fig. 10, we see a single-frequency oscillation of the intensity maxima, the latter moving almost in phase. This indeed corresponds to a low-wavelength lattice phonon, which becomes occupied for long enough times. As we discuss in Sec. V, the longest wavelength is of the order of the system size $L$, consistent with the almost in-phase oscillations of Fig. 10.

As we discussed in the previous section, lattice phonons have a finite gap. They can efficiently be excited in a quench experiment provided the energy available for collective excitations is large enough compared to $\Delta_{\mathrm{ph}}$ [see Eq. (11)].

\section{EXPERIMENTAL IMPLEMENTATION WITH ULTRACOLD BOSONS}

BECs with high densities and a controlled shape trapped in optical dipole traps are currently available in many laboratories. In principle, the setups normally employed are already very close to the one needed to study the crystallization effects presented in this work. In the following, we discuss the conditions needed to study our model in realistic experimental conditions, as well as the required parameter regime for observing the crystallization. Let us remark that the basic physics underlying the crystallization transition discussed here does not rely on the atoms being Bose condensed. This phenomenon could, in principle, also be observed with thermal clouds or fermionic gases.
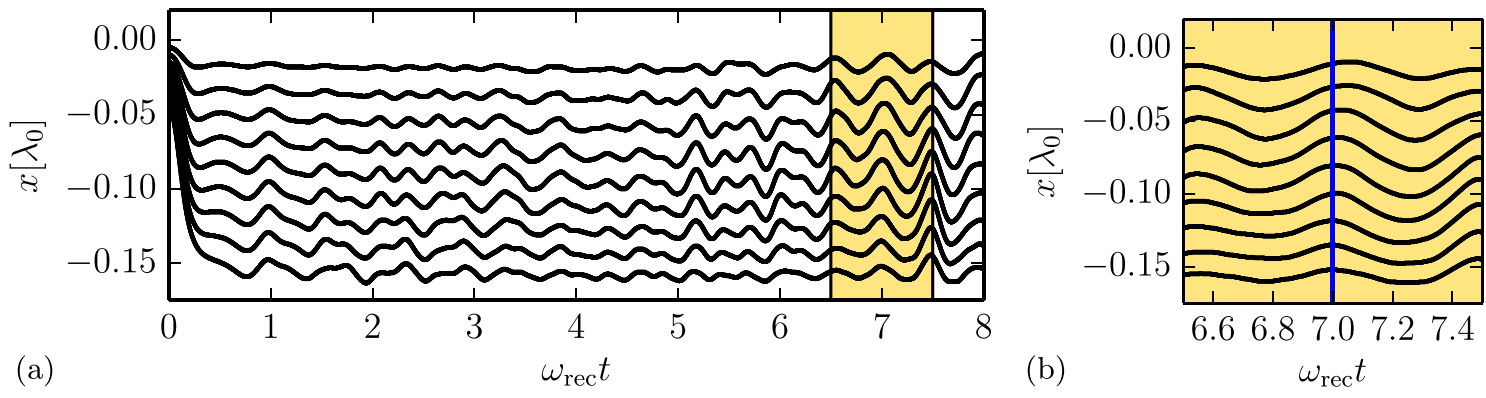

FIG. 10. Real-time evolution of the maxima of the intensity distribution as it is shown in Fig. 9. To simplify the comparison between the single curves, the maxima positions are shifted so that they all start at $x=0$. Panel (a) shows the total time evolution where one can clearly recognize collective phononlike excitations of the lattice after $\omega_{\text {rec }} t \gtrsim 5$. Panel (b) shows the zoom into the yellow marked area in (a) in order to demonstrate the slight dephasing between the oscillations of the maxima. All parameters are chosen as in Fig. 5. 
Apart from the fundamentally very interesting feature of supersolidity, the practical advantage of a BEC with respect to a thermal cloud resides in its high density and low temperature, both decreasing the required laser power. On the other hand, for degenerate Fermi gases, one could expect a strong dependence on the ratio between Fermi momentum and lattice constant [46-49].

We start by noting that using single-beam optical traps can also lead to heating instabilities but never generate a stationary lattice [17]. Similarly, operating very close to an atomic resonance has been shown to generate instabilities and a short-time formation of an optical lattice structure via so-called end-fire modes [16]. As this requires significant atomic excitation, it involves fast transverse acceleration with heating and destruction of the BEC. This is prevented in our model by an improved geometry and much larger atom-field detuning.

Our model Eqs. (1)-(3) is essentially 1D, which relies on the assumption that both the atoms and the light move and propagate essentially unidirectionally along $x$. In practice this can be implemented by using a transverse trapping of the atoms tight enough to freeze out the dynamics along $y$, $z$. With harmonic trapping potentials this amounts to the requirement that $\omega_{y, z}^{\text {ho }}$ is sufficiently larger than the BEC chemical potential $\mu$. Here, we still describe the onedimensional BEC using the GP equation, which requires the atom density to be large enough to be in the mean-field regime [39]. The enforcement of unidirectional propagation of light is more demanding since an appreciable amount of diffraction out of the BEC axis would be present inducing propagation also perpendicular to $x$. Apart from the use of hollow-core optical fibers around the BEC [50], one option available in many laboratories today is using a twodimensional array of tubes with spacing comparable with the wavelength of the light. This arrangement would generically produce destructive interference between the transverse field components diffracted from different tubes, so that if the latter are long enough, only the forward propagation along the tube axis would remain. In this configuration, each tube will act equally while the field propagates inside a medium with a refractive index given by the sum of the contributions from each tube. Indeed, since all tubes share the same backreflected field, there is a natural synchronization of the different tube lattices.

In any experimental realization a trap to confine the BEC along $x$ will also be present. In addition, the two laser intensities might differ to some extent due to experimental inaccuracies. As an exemplary case, we study the crystallization as in Sec. IV but add an harmonic trapping potential $V_{\text {ext }}(x)=\left(E_{\text {trap }} / 2\right) x^{2} / \lambda_{0}^{2}$ and choose different pump intensities $I_{l} \neq I_{r}$. It can be seen from Fig. 11 that the qualitative features of the crystalline phase remain the same as in the homogeneous case. The only difference is the parabolic envelope for the density as well as for the light intensity distribution and the shift of the distribution towards the
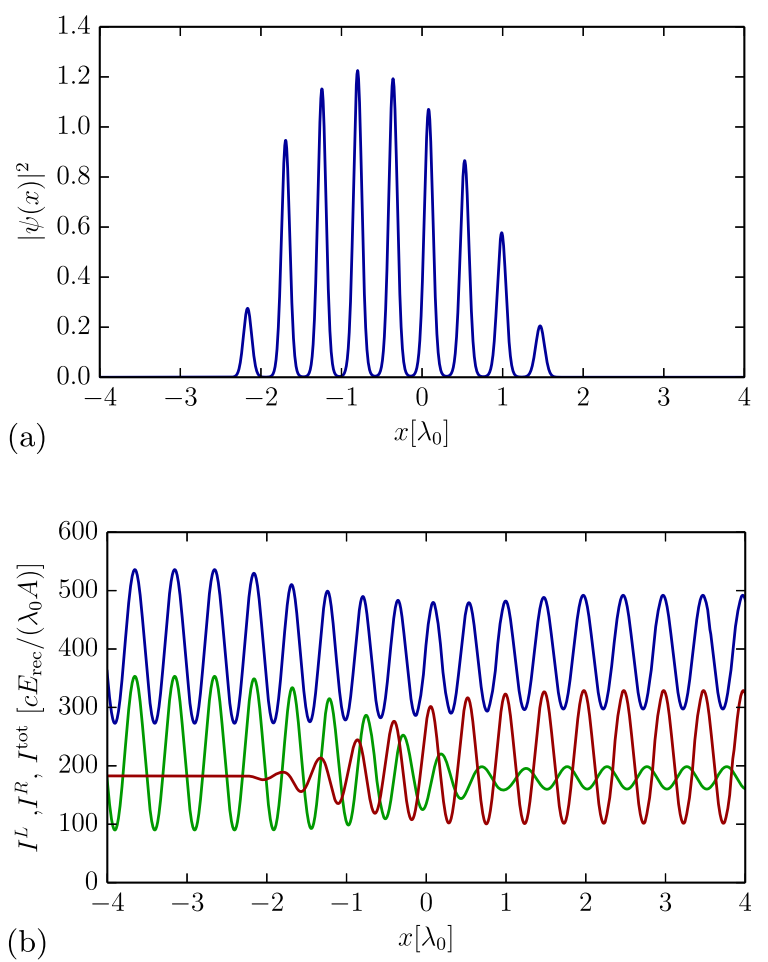

FIG. 11. (a) Crystal ground state and (b) corresponding intensity distribution for the field from left (green line) and right (red line) for the same parameters as in Fig. 5 with an additional external potential $V_{\text {ext }}(x)=\left(E_{\text {trap }} / 2\right) x^{2} / \lambda_{0}^{2}$ with $E_{\text {trap }}=1.0 E_{\text {rec }}$ and for different pump intensities from left and right $I_{l}=200$ and $I_{r}=150$. The solid blue line depicts the sum of both intensities.

direction of the higher intensity. The threshold behavior remains similar to the one we present in Fig. 4 with the only difference being an increase of the threshold intensity. A useful feature of the considered configuration is that the crystallization process can be observed in real time by looking at the amount of reflected light, since the transmitted part of the counterpropagating beam can be separated from the reflected part having orthogonal polarization.

In order to choose the most suitable atomic transition, pump detuning, and power, as well as BEC parameters like density and extension, one must consider the following constraints: we need to have (i) a low enough critical driving strength Eq. (9), which depends on the detuning $\Delta_{a}$ and spontaneous emission $\gamma$ through the real part of polarizability $\operatorname{Re} \alpha \sim \gamma / \Delta_{a}$, reading

$$
I_{c}^{L, R} \sim E_{\mathrm{rec}} \frac{\Delta_{a}^{2}}{\gamma^{2}} \frac{\lambda_{0}}{n_{A}}
$$

and at the same time (ii) a low enough BEC heating rate, which at the critical power reads

$$
\Gamma_{\text {heat }} \sim I_{c}^{L, R} \frac{\gamma^{2}}{\Delta_{a}^{2}} \sim E_{\text {rec }} \frac{\lambda_{0}}{n_{A}}
$$


with $n_{A}=N / A$ being the surface density of the medium with respect to the light propagation. From Eqs. (12) and (13) one sees that the crystallization is more easily achieved before the BEC is heated up if we increase the BEC surface density $n_{A}$. There is no favorable scaling either with detuning $\Delta_{a}$ or with the linewidth $\gamma$, since both heating rate Eq. (13) and critical power Eq. (12) scale with $\gamma^{2} / \Delta_{a}^{2}$. For commonly employed transitions like the $\mathrm{Rb}$ or Cs $D$ lines, the required laser power is easily achieved, but the heating rate can become a problem at too low densities due to the required laser powers and detunings. For instance, taking $N=10^{6}$ atoms confined over a transverse cross section $A \sim 5 \times 5 \mu \mathrm{m}^{2}$ and $\lambda_{0} \sim \mu \mathrm{m}$, we estimate a required power $I_{c} \sim \mathrm{W} / \mathrm{cm}^{2}$ with a heating rate $\Gamma_{\text {heat }} \sim 10 \mathrm{~Hz}$ for the rubidium 780-nm line with a detuning $\Delta_{a}=100 \mathrm{GHz}$ as well as for the cesium $D 2$ line with a detuning $\Delta_{a}=20 \mathrm{GHz}$. Such a heating rate still allows us to observe the crystal formation since, as we see in Fig. 9, this process takes place on the inverse recoil time scale, which is of the order of milliseconds.

\section{CONCLUSIONS AND OUTLOOK}

We predict that in suitable geometries roton instabilities originating from nonlinear free-space atom-light interactions can be tailored to generate stationary crystalline states. They involve an optical lattice showing an emergent spacing and phononic excitations, trapping the atoms at the intensity maxima.

The required translation invariant, mirror symmetric geometry can be realized using two orthogonal polarization degrees of freedom or frequency shifted counterpropagating beams. We estimate that the dynamics we study in this work should be accessible in already existing experimental setups on large quasi-1D Bose-Einstein condensates. Actually, in comparison with standard crossed beam dipole traps, one simply has to adapt and control the polarizations of the trapping lasers and choose suitable detunings. The ordering process should be easily observable not only by measuring the atomic distributions but directly by looking at the reflected light from the condensate. This nondestructive measurement allows for a real-time monitoring of the dynamics.

Our results open up an intriguing new direction in quantum simulations with ultracold atoms in optical lattices, where the latter are enriched by the presence of collective phononic excitations resulting from the spontaneous crystallization of light. In this spirit, the application of our approach to two dimensions and the inclusions of retardation effects as well as quantum fluctuations constitute the natural extension of this study.

\section{ACKNOWLEDGMENTS}

We thank S. Krämer for support in the numerical implementation and F. Meinert and T. Donner for helpful discussions on the experimental limitations and implementability of the system. We also thank J. Lang for useful discussions. We acknowledge support by the Austrian Science Fund FWF through projects SFB FoQuS P13 and I1697-N27. F.P. is supported by the APART program of the Austrian Academy of Science.

\section{APPENDIX A: CALCULATION OF THE EXCITATION SPECTRA}

Here, we describe in detail how the linearization of the Helmholtz and the GP equation leads to the collective excitation spectra below [see Eq. (8)] and above the threshold [see Fig. 6].

It is convenient to slightly rewrite the equations presented in Sec. II. Therefore, we define the relevant parameters of the system and useful units. We introduce the recoil energy $E_{\text {rec }}:=\hbar \omega_{\text {rec }}=\hbar^{2} k_{0}^{2} /(2 m)$ relative to the wave number $k_{0}=2 \pi / \lambda_{0}$ of the incoming lasers in vacuum. The dimensionless time is defined through the recoil frequency: $\tilde{t}:=\omega_{\text {rec }} t$. The dimensionless space variable is given in units of the incoming laser wavelength $\tilde{x}:=x / \lambda_{0}$. We also rescale the fields to have units of energy $\tilde{E}_{L, R}:=\sqrt{\alpha} E_{L, R} / \sqrt{A}$, and the atom-atom $s$-wave coupling to have units of energy times length $\tilde{g}_{\mathrm{c}}:=g_{\mathrm{c}} / A$. The GP equation [Eq. (1)] then reads

$$
\begin{aligned}
i \frac{\partial}{\partial \tilde{t}} \tilde{\psi}(\tilde{x}, \tilde{t})= & -\frac{1}{(2 \pi)^{2}} \frac{\partial^{2}}{\partial \tilde{x}^{2}} \tilde{\psi}(\tilde{x}, \tilde{t}) \\
& +\frac{V_{\mathrm{ext}}}{E_{\mathrm{rec}}} \tilde{\psi}(\tilde{x}, \tilde{t})-\frac{1}{E_{\mathrm{rec}}}\left[\left|\tilde{E}_{L}(\tilde{x})\right|^{2}\right. \\
& \left.+\left|\tilde{E}_{R}(\tilde{x})\right|^{2}\right] \tilde{\psi}(\tilde{x}, \tilde{t})+\frac{\tilde{g}_{c} N}{E_{\mathrm{rec}}}|\tilde{\psi}(\tilde{x}, \tilde{t})|^{2} \tilde{\psi}(\tilde{x}, \tilde{t}),
\end{aligned}
$$

and the Helmholtz equations [Eq. (3)] become

$$
\frac{\partial^{2}}{\partial \tilde{x}^{2}} \tilde{E}_{L, R}(\tilde{x})+(2 \pi)^{2}\left[1+\zeta|\tilde{\psi}(\tilde{x}, t)|^{2}\right] \tilde{E}_{L, R}(\tilde{x})=0 .
$$

Let us first consider the linearization of the Helmholtz equation [Eq. (A2)]. Inserting the ansatz already presented in Sec. III, namely, $\psi=\left(\psi_{0}+\delta \psi\right) e^{-i \mu t}$ and $E_{L, R}=$ $E_{L, R}^{(0)}+\delta E_{L, R}$, into Eq. (A2) and neglecting terms of second order leads to

$$
\begin{aligned}
& \partial_{x x} E_{L, R}^{(0)}+(2 \pi)^{2}\left[1+\zeta\left|\psi_{0}\right|^{2}\right] E_{L, R}^{(0)} \\
+ & \partial_{x x} \delta E_{L, R}+(2 \pi)^{2}\left[1+\zeta\left|\psi_{0}\right|^{2}\right] \delta E_{L, R} \\
+ & (2 \pi)^{2} \zeta\left[\psi_{0} \delta \psi^{*}+\delta \psi \psi_{0}^{*}\right] E_{L, R}^{(0)}=0 .
\end{aligned}
$$

The first line [Eq. (A3)] corresponds to the Helmholtz equation for the steady state $E_{L, R}^{(0)}$ and, therefore, it is equal to zero. The second line [Eq. (A4)] is the Helmholtz equation for the field perturbation, whereas the third line 
[Eq. (A5)] describes the linear coupling between the field and the BEC.

This equation can be rewritten in the following form:

$$
\left(M+K_{\mathrm{eff}}^{2}\right) \cdot \delta \mathbf{E}_{L, R}=-(2 \pi)^{2} \zeta \mathcal{E}_{L, R}^{(0)} \cdot\left(\Psi_{0} \cdot \delta \boldsymbol{\psi}^{*}+\text { H.c. }\right),
$$

where we define the matrices

$$
\begin{aligned}
M\left(x, x^{\prime}\right) & :=\partial_{x x} \delta\left(x-x^{\prime}\right), \\
K_{\mathrm{eff}}^{2}\left(x, x^{\prime}\right) & :=(2 \pi)^{2}\left[1+\zeta n_{0}(x)\right] \delta\left(x-x^{\prime}\right), \\
\mathcal{E}_{L, R}^{(0)}\left(x, x^{\prime}\right) & :=E_{L, R}^{(0)} \delta\left(x-x^{\prime}\right), \\
\Psi_{0}\left(x, x^{\prime}\right) & :=\psi_{0} \delta\left(x-x^{\prime}\right),
\end{aligned}
$$

and the scalar product

$$
M \cdot \mathbf{f}=\int d x^{\prime} M\left(x, x^{\prime}\right) f\left(x^{\prime}\right)
$$

The formal solution of the linearized Helmholtz equation [Eq. (A6)] is

$$
\delta \mathbf{E}_{L, R}=-(2 \pi)^{2} \zeta\left(M+K_{\text {eff }}^{2}\right)^{-1} \cdot \mathcal{E}_{L, R}^{(0)} \cdot\left(\Psi_{0} \cdot \boldsymbol{\psi}^{*}+\text { H.c. }\right) .
$$

The linearization of the Gross-Pitaevski equation [Eq. (A1)] follows a similar procedure as presented above. Performing the same ansatz and neglecting the secondorder terms leads to

$$
\begin{aligned}
i \partial_{t} \delta \psi+\mu \delta \psi= & -\frac{1}{(2 \pi)^{2}} \partial_{x x} \delta \psi-\frac{1}{E_{\text {rec }}}\left[\psi_{0}\left(E_{L}^{(0)^{*}} \delta E_{L}+E_{R}^{(0)^{*}} \delta E_{R}+\text { c.c. }\right)+\delta \psi\left(\left|E_{L}^{(0)}\right|^{2}+\left|E_{R}^{(0)}\right|^{2}\right)\right] \\
& +\frac{g_{c} N}{E_{\text {rec }}}\left[\left|\psi_{0}\right|^{2} \delta \psi+\psi_{0}\left(\psi_{0}^{*} \delta \psi+\psi_{0} \delta \psi^{*}\right)\right]-\psi_{0} \mu \\
& -\frac{1}{(2 \pi)^{2}} \partial_{x x} \psi_{0}-\frac{1}{E_{\mathrm{rec}}} \psi_{0}\left(\left|E_{L}^{(0)}\right|^{2}+\left|E_{R}^{(0)}\right|^{2}\right)+\frac{g_{c} N}{E_{\mathrm{rec}}}\left|\psi_{0}\right|^{2} \psi_{0} .
\end{aligned}
$$

The last line of Eq. (A13) corresponds to the stationary GP equation and, therefore, it vanishes, as it defines how the chemical potential is related to the field amplitude and the particle-particle interaction $g_{c}$, namely via

$$
\mu=\frac{g_{c} N}{L E_{\mathrm{rec}}}-\frac{2\left|E_{L, R}^{(0)}\right|^{2}}{E_{\mathrm{rec}}} .
$$

Inserting the formal solution Eq. (A12) into the linearized GP equation [Eq. (A13)] and performing a Fourier transform via $f(x)=\frac{1}{\sqrt{L}} \sum_{k} e^{i k x} f(k)$ and $M\left(x, x^{\prime}\right)=$ $\frac{1}{L} \sum_{k, k^{\prime}} e^{i k x} e^{i k^{\prime} x^{\prime}} M\left(k, k^{\prime}\right)$ gives

$$
\begin{aligned}
i \partial_{t} \rrbracket \boldsymbol{\psi}= & \left(-\mu \rrbracket+T+A_{L}+\tilde{A}_{L}+A_{R}+\tilde{A}_{R}+I_{\mathrm{tot}}+2 \nu_{0}\right) \boldsymbol{\psi} \\
& +\left(A_{L}+\tilde{A}_{L}+A_{R}+\tilde{A}_{R}+\nu_{0}\right) \mathcal{P} \boldsymbol{\psi}^{*},
\end{aligned}
$$

where $\mathbb{1}$ denotes the identity matrix and $\mathcal{P}$ is the parity operator, i.e., $\mathcal{P} \boldsymbol{\psi}(k)=\boldsymbol{\psi}(-k)$. We define the following matrices:

$$
\begin{aligned}
T\left(k, k^{\prime}\right) & :=\frac{k^{2}}{(2 \pi)^{2}} \delta\left(k-k^{\prime}\right), \\
\mathcal{I}_{\text {tot }}\left(k, k^{\prime}\right) & :=-\frac{1}{E_{\text {rec }} \sqrt{L}} I_{\text {tot }}\left(k-k^{\prime}\right), \\
\nu_{0}\left(k, k^{\prime}\right) & :=\frac{g_{c} N}{E_{\text {rec }} \sqrt{L}} n_{0}\left(k-k^{\prime}\right),
\end{aligned}
$$

$$
\begin{aligned}
& A_{L, R}\left(k, k^{\prime}\right):= \\
& \quad \frac{\zeta \pi^{2}}{E_{\mathrm{rec}} L} \sum_{k_{1}, k_{2}} V_{L, R}^{\dagger}\left(k, k_{1}\right) Q^{-1}\left(k_{1}, k_{2}\right) V_{L, R}\left(k_{2}, k^{\prime}\right), \\
& \tilde{A}_{L, R}\left(k, k^{\prime}\right):= \\
& \quad \frac{\zeta \pi^{2}}{E_{\mathrm{rec}} L} \sum_{k_{1}, k_{2}} V_{L, R}\left(k, k_{1}\right) Q^{-1}\left(k_{1}, k_{2}\right) V_{L, R}^{\dagger}\left(k_{2}, k^{\prime}\right),
\end{aligned}
$$

where $I_{\text {tot }}(k)$ and $n_{0}(k)$ are the Fourier transforms of the total intensity distribution and the BEC density. We also define the additional matrices $V_{L, R}\left(k, k^{\prime}\right):=$ $\sum_{k^{\prime \prime}} \psi_{0}^{*}\left(k^{\prime \prime}\right) E_{L, R}^{(0)}\left(k^{\prime \prime}+k-k^{\prime}\right) \quad$ and $\quad Q\left(k, k^{\prime}\right):=-k^{2} \delta(k-$ $\left.k^{\prime}\right)+1 / \sqrt{L} k_{\mathrm{eff}}^{2}\left(k-k^{\prime}\right)$, where $k_{\mathrm{eff}}^{2}(k)$ is the Fourier transform of $(2 \pi)^{2}\left[1+\zeta n_{0}(x)\right]$. In the following, we call the sum of the $A$ matrices $\mathcal{A}\left(k, k^{\prime}\right):=A_{L}\left(k, k^{\prime}\right)+\tilde{A}_{L}\left(k, k^{\prime}\right)+$ $A_{R}\left(k, k^{\prime}\right)+\tilde{A}_{R}\left(k, k^{\prime}\right)$.

Let us now define the spinor $\Psi(q):=\left(\psi(q), \psi^{*}(q)\right)^{T}$, where $\psi(q)$ defines a single momentum component of $\boldsymbol{\psi}$ from Eq. (A15). This definition allows us to write the GP equation in the form $i \partial_{t} \Psi(q)=\sum_{q^{\prime}} R\left(q, q^{\prime}\right) \Psi\left(q^{\prime}\right)$, where the matrix $R$ is defined as follows: 
$R\left(q, q^{\prime}\right)=\left(\begin{array}{cc}-\mu \delta\left(q-q^{\prime}\right)+\frac{q^{2}}{(2 \pi)^{2}} \delta\left(q-q^{\prime}\right) \mathcal{I}_{\text {tot }}\left(q-q^{\prime}\right)+\mathcal{A}\left(q, q^{\prime}\right) & \nu_{0}\left(q, q^{\prime}\right)+\mathcal{A}\left(q, q^{\prime}\right) \\ -\nu_{0}\left(q, q^{\prime}\right)-\mathcal{A}\left(-q,-q^{\prime}\right) & -\left[-\mu \delta\left(q-q^{\prime}\right)+\frac{q^{2}}{(2 \pi)^{2}} \delta\left(q-q^{\prime}\right) \mathcal{I}_{\mathrm{tot}}\left(q-q^{\prime}\right)+\mathcal{A}\left(q, q^{\prime}\right)\right]\end{array}\right)$

This equation now enables us to calculate the excitation spectrum of the considered system for any arbitrary intensity and BEC density distribution by calculating the eigenvalues of the matrix $R$.

\section{Collective spectrum in the homogeneous phase}

If we now use the ansatz already presented in Sec. III, namely, $\psi_{0}(x, t)=1 / \sqrt{L}$ and $E_{L, R}^{(0)}=C \exp \left( \pm i k_{\mathrm{eff}} x\right)$, we can calculate the excitation spectrum below threshold. This ansatz implies $I_{\text {tot }}(x)=\left|E_{R}^{(0)}\right|^{2}+\left|E_{L}^{(0)}\right|^{2}=2|C|^{2}$ and $n_{0}(x)=1 / L$, which results in

$$
\begin{gathered}
\mathcal{I}_{\text {tot }}(k)=-\frac{8|C|^{2}}{E_{\mathrm{rec}}} \delta(k), \\
\nu_{0}(k)=\frac{g_{c} N}{E_{\mathrm{rec}} L} \delta(k) .
\end{gathered}
$$

In addition, the matrices $Q$ and $V$ amount to

$$
\begin{gathered}
Q(k)=\left(k_{\mathrm{eff}}^{2}-k^{2}\right) \delta(k), \\
V_{L}(k)=C \sqrt{L} \delta\left(k+k_{\mathrm{eff}}\right),
\end{gathered}
$$

resulting in

$A_{L, R}\left(q, q^{\prime}\right)=-\frac{\zeta}{E_{\mathrm{rec}}} \frac{|C|^{2}(2 \pi)^{2}}{L} \frac{1}{q^{2} \mp 2 k_{\mathrm{eff}} q} \delta\left(q-q^{\prime}\right)$.

Note that in this special case, $\tilde{A}_{L, R}=A_{L, R}$. If one now calculates the matrix $R$ via Eq. (A23) and solves $\operatorname{det}\left[R\left(q-q^{\prime}\right)-\omega \mathbb{1}\right]=0$, one gets

$\omega^{2}-\frac{q^{2}}{(2 \pi)^{2}}\left[\frac{q^{2}}{2 m}+2 \frac{g_{c} N}{E_{\mathrm{rec}} L}-\frac{8 \zeta}{E_{\mathrm{rec}}} \frac{|C|^{2}(2 \pi)^{2}}{L} \frac{1}{q^{2}-4 k_{\mathrm{eff}}^{2}}\right]=0$.

Transforming this equation back into the original units leads to the excitation spectrum [Eq. (8)] presented in Sec. III.

\section{Collective spectrum above threshold}

Let us now move on to the calculation of the collective excitation spectrum above threshold as it is presented in Sec. V. In this case, an analytical answer like the one presented in the previous section is not possible, since the translation invariance is broken so that the matrices describing the linear system are not diagonal in momentum space. Therefore, a numerical approach is required, involving, in general, the discretization of the position (momentum) continuum.

The matrices defined in Eqs. (A16)-(A22) can be calculated by numerically finding the Fourier transforms of the stationary states found via complex time evolution in Sec. IV. The resulting total matrix $R$ can then be diagonalized numerically.

A further difficulty arising in our setup is that in the stationary crystalline solution, the total light intensity and atom density are periodic, whereas the intensity of each polarization component is not. This originates from the repeated reflection from the density grating, introducing the decaying envelope shown in Fig. (5b) of the main text. This prevents the use of the quasimomentum to label the excitation modes. Therefore, we use the momentum corresponding to the largest component of the eigenvector in order to order the eigenvalues in Fig. 6.

\section{APPENDIX B: NUMERICAL METHODS}

The model described in Sec. II constitutes a coupled system of equations [Eqs. (1) and (3)]. In this appendix, we briefly discuss the numerical methods we use to simulate the time evolution of the studied system as it is used in Secs. IV-VII.

The algorithm consists of two parts. First, we need to solve the Helmholtz equation [Eq. (3)] for a given spacedependent susceptibility Eq. (4). This corresponds to an initial value problem with the boundary conditions

$$
\begin{gathered}
E(x=-L / 2)=A_{L}+B_{L} \\
\partial_{x} E(x=-L / 2)=i k_{0}\left(A_{L}-B L\right) .
\end{gathered}
$$

Here, $A_{L}$ and $B_{L}$ define the incoming $\left(A_{L}\right)$ and outgoing $\left(B_{L}\right)$ field amplitudes at the left side of the BEC. They are related to the amplitudes on the right side via

$$
\begin{aligned}
& B_{L}=R A_{L}+T D_{R}, \\
& C_{R}=T A_{l}+R D_{R},
\end{aligned}
$$

with the system's reflection and transmission coefficients $R$ and $T$. Of course, these reflection and transmission coefficients depend on the system's susceptibility. They can easily be estimated by solving the Helmholtz $(\mathrm{HH})$ equation 
for an arbitrary initial condition [Eqs. (B1) and (B2)], leading to well-defined fields at the boundaries allowing for an estimation of the right-hand amplitudes $C_{R}$ and $D_{R}$. Hence, $R$ and $T$ can be calculated via Eqs. (B3) and (B4). As soon as we know the initial conditions we can find the solution of the Helmholtz equation via spatial integration performed by a fourth-order Runge-Kutta solver.

The solution of the HH equation is then used to calculate the optical potential [Eqs. (5)]. The time evolution of the GP equation with the newly found potential is then calculated by using a split step method. Note that the $\mathrm{HH}$ equation has to be solved within each time step, resulting in a modified potential for the next time step in the GP equation. The time evolution is finished as soon as the system is found in a stationary state.

[1] A. V. Andreev, V. I. Emel'yanov, and Y. A. Il'inski, Collective Spontaneous Emission (Dicke Superradiance), Phys. Usp. 23, 493 (1980).

[2] I. B. Mekhov, C. Maschler, and H. Ritsch, Light Scattering from Ultracold Atoms in Optical Lattices as an Optical Probe of Quantum Statistics, Phys. Rev. A 76, 053618 (2007).

[3] H. Zoubi and H. Ritsch, Metastability and Directional Emission Characteristics of Excitons in $1 D$ Optical Lattices, Europhys. Lett. 90, 23001 (2010).

[4] A. Braun, G. Korn, X. Liu, D. Du, J. Squier, and G. Mourou, Self-Channeling of High-Peak-Power Femtosecond Laser Pulses in Air, Opt. Lett. 20, 73 (1995).

[5] D. Kartashov, S. Ališauskas, A. Pugžlys, A. Voronin, A. Zheltikov, M. Petrarca, P. Béjot, J. Kasparian, J.-P. Wolf, and A. Baltuška, Mid-Infrared Laser Filamentation in Molecular Gases, Opt. Lett. 38, 3194 (2013).

[6] A. Zheltikov, A. L'Huillier, and F. Krausz, Springer Handbook of Lasers and Optics (Springer, New York, 2012) pp. 161-251.

[7] W. Singer, M. Frick, S. Bernet, and M. Ritsch-Marte, Self-Organized Array of Regularly Spaced Microbeads in a Fiber-Optical Trap, J. Opt. Soc. Am. B 20, 1568 (2003).

[8] M. M. Burns, J.-M. Fournier, and J. A. Golovchenko, Optical Matter: Crystallization and Binding in Intense Optical Fields, Science 249, 749 (1990).

[9] S. A. Tatarkova, A.E. Carruthers, and K. Dholakia, One-Dimensional Optically Bound Arrays of Microscopic Particles, Phys. Rev. Lett. 89, 283901 (2002).

[10] V. Demergis and E.-L. Florin, Ultrastrong Optical Binding of Metallic Nanoparticles, Nano Lett. 12, 5756 (2012).

[11] V. Karásek, T. Čižmár, O. Brzobohatý, P. Zemánek, V. Garcés-Chávez, and K. Dholakia, Long-Range OneDimensional Longitudinal Optical Binding, Phys. Rev. Lett. 101, 143601 (2008).

[12] V. Karásek, O. Brzobohatý, and P. Zemánek, Longitudinal Optical Binding of Several Spherical Particles Studied by the Coupled Dipole Method, J. Opt. A 11, 034009 (2009).

[13] K. Dholakia and P. Zemánek, Colloquium: Gripped by Light: Optical Binding, Rev. Mod. Phys. 82, 1767 (2010).
[14] R. Bonifacio and L. De Salvo, Collective Atomic Recoil Laser (CARL) Optical Gain without Inversion by Collective Atomic Recoil and Self-Bunching of Two-Level Atoms, Nucl. Instrum. Methods Phys. Res., Sect. A 341, 360 (1994).

[15] M. Saffman, Self-Induced Dipole Force and Filamentation Instability of a Matter Wave, Phys. Rev. Lett. 81, 65 (1998).

[16] S. Inouye, A. P. Chikkatur, D. M. Stamper-Kurn, J. Stenger, D. E. Pritchard, and W. Ketterle, Superradiant Rayleigh Scattering from a Bose-Einstein Condensate, Science 285, 571 (1999).

[17] N. Piovella, R. Bonifacio, B. W. J. McNeil, and G. R. M. Robb, Superradiant Light Scattering and Grating Formation in Cold Atomic Vapours, Opt. Commun. 187, 165 (2001).

[18] D. H. J. O’Dell, S. Giovanazzi, and G. Kurizki, Rotons in Gaseous Bose-Einstein Condensates Irradiated by a Laser, Phys. Rev. Lett. 90, 110402 (2003).

[19] Y. Yoshikawa, Y. Torii, and T. Kuga, Superradiant Light Scattering from Thermal Atomic Vapors, Phys. Rev. Lett. 94, 083602 (2005).

[20] G. A. Muradyan, Y. Wang, W. Williams, and M. Saffman, Nonlinear Guided Waves and Their Applications (Optical Society of America, Washington, DC, 2005).

[21] S. Slama, S. Bux, G. Krenz, C. Zimmermann, and P. W. Courteille, Superradiant Rayleigh Scattering and Collective Atomic Recoil Lasing in a Ring Cavity, Phys. Rev. Lett. 98, 053603 (2007).

[22] J. A. Greenberg, B. L. Schmittberger, and D. J. Gauthier, Bunching-Induced Optical Nonlinearity and Instability in Cold Atoms, Opt. Express 19, 22535 (2011).

[23] B. L. Schmittberger and D. J. Gauthier, Spontaneous Emergence of Free-Space Optical and Atomic Patterns, arXiv:1603.06294.

[24] G. Labeyrie, E. Tesio, P. M. Gomes, G.-L. Oppo, W. J. Firth, G. R. M. Robb, A. S. Arnold, R. Kaiser, and T. Ackemann, Optomechanical Self-Structuring in a Cold Atomic Gas, Nat. Photonics 8, 321 (2014).

[25] G. R. M. Robb, E. Tesio, G.-L. Oppo, W. J. Firth, T. Ackemann, and R. Bonifacio, Quantum Threshold for Optomechanical Self-Structuring in a Bose-Einstein Condensate, Phys. Rev. Lett. 114, 173903 (2015).

[26] P. Domokos and H. Ritsch, Collective Cooling and SelfOrganization of Atoms in a Cavity, Phys. Rev. Lett. 89, 253003 (2002).

[27] A. T. Black, H. W. Chan, and V. Vuletić, Observation of Collective Friction Forces due to Spatial Self-Organization of Atoms: From Rayleigh to Bragg Scattering, Phys. Rev. Lett. 91, 203001 (2003).

[28] K. J. Arnold, M. P. Baden, and M.D. Barrett, SelfOrganization Threshold Scaling for Thermal Atoms Coupled to a Cavity, Phys. Rev. Lett. 109, 153002 (2012).

[29] H. Ritsch, P. Domokos, F. Brennecke, and T. Esslinger, Cold Atoms in Cavity-Generated Dynamical Optical Potentials, Rev. Mod. Phys. 85, 553 (2013).

[30] K. Baumann, C. Guerlin, F. Brennecke, and T. Esslinger, Dicke Quantum Phase Transition with a Superfluid Gas in an Optical Cavity, Nature (London) 464, 1301 (2010).

[31] H. Keßler, J. Klinder, M. Wolke, and A. Hemmerich, Steering Matter Wave Superradiance with an UltranarrowBand Optical Cavity, Phys. Rev. Lett. 113, 070404 (2014). 
[32] S. Ostermann, T. Grießer, and H. Ritsch, Atomic SelfOrdering in a Ring Cavity with Counterpropagating Pump Fields, Europhys. Lett. 109, 43001 (2015).

[33] R. Mottl, F. Brennecke, K. Baumann, R. Landig, T. Donner, and T. Esslinger, Roton-Type Mode Softening in a Quantum Gas with Cavity-Mediated Long-Range Interactions, Science 336, 1570 (2012).

[34] T. Grießer and H. Ritsch, Light-Induced Crystallization of Cold Atoms in a 1D Optical Trap, Phys. Rev. Lett. 111, 055702 (2013).

[35] D. E. Chang, J. Ignacio Cirac, and H. J. Kimble, SelfOrganization of Atoms along a Nanophotonic Waveguide, Phys. Rev. Lett. 110, 113606 (2013).

[36] S. Gopalakrishnan, B. L. Lev, and P. M. Goldbart, Emergent Crystallinity and Frustration with Bose-Einstein Condensates in Multimode Cavities, Nat. Phys. 5, 845 (2009).

[37] D. E. Chang, V. Gritsev, G. Morigi, V. Vuletić, M. D. Lukin, and E. A. Demler, Crystallization of Strongly Interacting Photons in a Nonlinear Optical Fibre, Nat. Phys. 4, 884 (2008).

[38] J. Otterbach, M. Moos, D. Muth, and M. Fleischhauer, Wigner Crystallization of Single Photons in Cold Rydberg Ensembles, Phys. Rev. Lett. 111, 113001 (2013).

[39] S. Stringari and L. Pitaevskii, Bose-Einstein Condensation (Oxford University Press, New York, 2003).

[40] P. M. Chaikin and T. C. Lubensky, Principles of Condensed Matter Physics (Cambridge University Press, Cambridge, England, 2000).

[41] S. Ostermann, M. Sonnleitner, and H. Ritsch, Scattering Approach to Two-Colour Light Forces and Self-Ordering of Polarizable Particles, New J. Phys. 16, 043017 (2014).

[42] I. H. Deutsch, R. J. C. Spreeuw, S. L. Rolston, and W. D. Phillips, Photonic Band Gaps in Optical Lattices, Phys. Rev. A 52, 1394 (1995).

[43] N. W. Ashcroft and N. David Mermin, Solid State Physics, Books/Cole Cengage Learning (Inc, 1976).

[44] E. Wigner, Effects of the Electron Interaction on the Energy Levels of Electrons in Metals, Trans. Faraday Soc. 34, 678 (1938).

[45] V. I. Yukalov and K. Ziegler, Instability of Insulating States in Optical Lattices due to Collective Phonon Excitations, Phys. Rev. A 91, 023628 (2015).

[46] J. Keeling, M. J. Bhaseen, and B. D. Simons, Fermionic Superradiance in a Transversely Pumped Optical Cavity, Phys. Rev. Lett. 112, 143002 (2014).

[47] F. Piazza and P. Strack, Umklapp Superradiance with a Collisionless Quantum Degenerate Fermi Gas, Phys. Rev. Lett. 112, 143003 (2014).

[48] Y. Chen, Z. Yu, and H. Zhai, Superradiance of Degenerate Fermi Gases in a Cavity, Phys. Rev. Lett. 112, 143004 (2014).

[49] R. M. Sandner, W. Niedenzu, F. Piazza, and H. Ritsch, SelfOrdered Stationary States of Driven Quantum Degenerate Gases in Optical Resonators, Europhys. Lett. 111, 53001 (2015).

[50] C. A. Christensen, S. Will, M. Saba, G.-B. Jo, Y.-I. Shin, W. Ketterle, and D. Pritchard, Trapping of Ultracold Atoms in a Hollow-Core Photonic Crystal Fiber, Phys. Rev. A 78, 033429 (2008). 